\title{
Dickkopf-3 as a new potential marker for neoangiogenesis in colorectal cancer: Expression in cancer tissue and adjacent non-cancerous tissue ${ }^{1}$
}

\author{
Matthias Zitt ${ }^{\mathrm{a}, *}$, Gerold Untergasser ${ }^{\mathrm{b}}$, Albert Amberger ${ }^{\mathrm{c}}$, Patrizia Moser ${ }^{\mathrm{d}}$, Sylvia Stadlmann ${ }^{\mathrm{e}}$, \\ Marion Zitt ${ }^{\mathrm{c}}$, Hannes M. Müller ${ }^{\mathrm{a}}$, Gilbert Mühlmann ${ }^{\mathrm{a}}$, Alexander Perathoner ${ }^{\mathrm{a}}$, Raimund Margreiter ${ }^{\mathrm{a}, \mathrm{c}}$, \\ Eberhard Gunsilius ${ }^{\mathrm{b}}$ and Dietmar Öfner ${ }^{\mathrm{a}}$ \\ ${ }^{a}$ Department of General and Transplant Surgery, Innsbruck Medical University, Austria \\ ${ }^{\mathrm{b}}$ Laboratory of Tumor Biology \& Angiogenesis, Department of Hematology and Oncology, Innsbruck Medical \\ University, Austria \\ ${ }^{\mathrm{c}}$ Tyrolean Cancer Research Institute, Innsbruck, Austria \\ ${ }^{\mathrm{d}}$ Department of Pathology, Innsbruck Medical University, Austria \\ e Department of Pathology, Basel University Hospital, Switzerland
}

\begin{abstract}
Gene expression of Dickkopf-3 (Dkk-3) has been shown to be upregulated in tumor endothelium of colorectal cancer (CRC). For the first time, we analyzed Dkk-3 protein expression in CRC and its potential as a marker for neoangiogenesis. We used tissue microarrays (TMAs) to investigate Dkk-3 in microvessels of 403 CRC samples, 318 appropriate adjacent non-cancerous samples and 127 normal colorectal samples. Of cancer samples with CD31-positive microvessels, 67.7\% were positive for Dkk-3. Dkk-3 staining was demonstrated in endothelial cells of all microvessels in nearly all cases. Dkk-3-positive samples showed a higher mean microvessel count than did Dkk-3-negative samples $(P=0.001)$. Dkk-3 expression increased with rising numbers of microvessels per sample $(P<0.0001)$. In adjacent samples with CD31-positive microvessels, 56\% were Dkk-3-positive in all microvessels. Similar to cancer samples, Dkk-3-positive adjacent samples had a higher mean microvessel count than did Dkk-3-negative samples $(P<0.0001)$, and Dkk-3 expression also increased with rising numbers of microvessels $(P<0.0001)$. All microvessels in normal mucosa samples were negative for Dkk-3.

Dkk-3 can be considered a putative pro-angiogenic protein in neovascularization and may possibly be a marker for neoangiogenesis in CRC. Further investigations will elucidate whether Dkk-3 is a target structure for novel therapies.
\end{abstract}

Keywords: Dickkopf-3, neoangiogenesis, colorectal cancer, adjacent tissue, tissue microarrays

\section{Introduction}

Advances in multidisciplinary treatment and intensive follow-up programs have improved the outcome

\footnotetext{
${ }^{1}$ Grant: Österreichische Krebshilfe-Krebsgesellschaft Tirol 2006.

* Corresponding author: Matthias Zitt, MD, Department of General and Transplant Surgery, Innsbruck Medical University, Anichstrasse 35, A-6020 Innsbruck, Austria. Tel.: +43 512504 0; Fax: +43 512504 28519; E-mail: matthias.zitt@i-med.ac.at.
}

of colorectal cancer patients [1-3]. However, tumor invasion, lymphatic spread and distant metastasis still limit the prognosis of colorectal cancer. Neoangiogenesis is a hallmark of most malignancies, and inhibition of this process is considered a promising strategy for cancer treatment. Folkman first mentioned that tumor progression is dependent on the development of new blood vessels [4]. Environmental and genetic alterations induce an "angiogenic switch" during tumor growth, with either upregulation of angiogenic or 
downregulation of antiangiogenic factors [5,6]. Several differences between tumor-associated endothelium in abnormal microvessels and normal endothelium have been established by studies of their structure and function [7]. St. Croix et al. revealed 46 genes showing stronger expression in tumor endothelium of colorectal cancer tissues than in endothelium of normal colonic mucosa. These genes, termed tumor endothelial markers (TEMs), show that tumor-associated and normal endothelium differ at the molecular level [8].

Dickkopf-3 (Dkk-3) - one of these TEMs - has been isolated according to its sequence homology to the cysteine-rich Dickkopf family of secreted canonical Wnt-inhibitors. The human Dickkopf (hDkk) family includes Dkk-1, Dkk-2, Dkk-3, Dkk-4 and a unique Dkk-3-related protein called Soggy (Sgy) [9]. Human Dkks 1-4 are glycoproteins of 255-350 amino acids that contain a signal sequence and share two conserved cysteine-rich domains, each of which displays a characteristic spacing of cysteines and other conserved amino acids. The N-terminal cysteine-rich domain - Dkk N (formerly termed Cys1) - is unique to the Dkks, while the C-terminal cysteine-rich domain - formerly termed Cys 2 - has a pattern of ten cysteines related to that of the colipase family. These two domains are separated by a non-conserved linker region [10]. Dkk-3 is the most divergent of the four human Dkks and possesses an extended $\mathrm{N}$-terminal domain, that precedes the Cys1 domain, and an extended $\mathrm{C}$-terminal region, that follows Cys-2 [9]. Dkk-1 and Dkk-4 have been found to inhibit Wnt signaling by binding to the transmembrane receptors Kremen 1 and $2(\mathrm{Krm} 1 / 2)$ and lipoprotein receptor-related protein 5/6 (LRP5/6), a coreceptor of the $\mathrm{Wnt} / \mathrm{Fz}$ receptor $[10,11]$. In contrast, the biological function and interaction partners of Dkk-3 remain unclear. There is no evidence that Dkk-3 is involved in inhibition of the WNT signaling pathway and thus the beta-catenin destabilization process [11]. However, gene expression of Dkk-3 has been shown to be upregulated in tumor endothelium of colorectal cancer tissue [8].

To our best knowledge, Dkk-3 protein expression in tumor microvessels has not been investigated in colorectal cancer so far. Thus, the current study was designed to analyze the role of Dkk-3 as a potential factor in neoangiogenesis in colorectal cancer. For a highthroughput approach we employed tissue microarrays (TMAs) containing a large series of colorectal cancer tissue and adjacent non-cancerous tissue.

\section{Materials and methods}

\subsection{Specimens and tissue microarray (TMA) construction}

Colorectal cancer specimens $(n=403)$ and corresponding colorectal adjacent non-cancerous tissue specimens $(n=318)$ from patients who underwent oncologic resection of colorectal cancer at Innsbruck Medical University were utilized for the study. The 403 patients were treated between 1992 and 2001 [224 men $(55.6 \%)$ and 179 women $(44.4 \%)$ at a mean age of 69.1 years (95\% CI: 67.9-70.2)]. Resected tumors were histopathologically examined according to the guidelines of the Union Internationale Contre le Cancer (UICC) and were graded according to WHO classification. Chemotherapy was recommended to patients with UICC stage III/IV. Demographic data of all patients were recruited from our computerized surgical documentation system for colorectal cancer (ChiBASE). All patients were subsequently followed up as described elsewhere [3].

Formalin-fixed and paraffin-embedded specimens and corresponding hematoxylin-erythrosin (HE) staining sections were obtained from the Department of Pathology. Firstly, HE staining sections were observed and representative areas of cancer and adjacent non-cancerous tissue marked. Then tissue cylinders ( $0.6 \mathrm{~mm}$ in diameter) were punched from the corresponding region of paraffin samples using a custommade precision instrument (Beecher Instruments, Silver Spring, MD). The empty TMA paraffin block $(25 \mathrm{~mm} \times 35 \mathrm{~mm})$ was holed through Tissue Arrayer ( $0.6 \mathrm{~mm}$ in diameter), and the tissue cylinders were neatly inserted in these holes. A different kind of tissue was also inserted as a marker, namely spleen tissue in this study. Each TMA paraffin block was sliced into 3- $\mu \mathrm{m}$-thick sections that were transferred to glass slides using the Paraffin Sectioning Aid System (Instrumedics, Hackensack, NJ).

In addition, a tissue microarray (Normal colon/ rectum tissue multi-sites tissue microarray; diameter: $1.5 \mathrm{~mm}$; thickness: $5 \mu \mathrm{m}$ ) with normal colorectal mucosal specimens ( $n=127$; male/female: $n=82 / 45$; colon/rectum: $n=58 / 69$ ) were purchased from US Biomax Inc. (Rockville, MD, USA).

\subsection{Immunohistochemistry}

TMA paraffin sections (cancer tissue sections, adjacent non-cancerous tissue sections and normal col- 
orectal mucosa sections) were deparaffinized and rehydrated with xylene and graded alcohol series. Thereafter, antigens were retrieved by microwave treatment in citrate-buffer (10 mM, pH 6.0) and endogenous peroxidase activity was blocked with $3 \% \mathrm{H}_{2} \mathrm{O}_{2} /$ methanol. Sections were incubated for $45 \mathrm{~min}$ in blocking solution containing $10 \%$ bovine calf serum (Dako Cytomation, Copenhagen, Denmark) and then stained for $1 \mathrm{~h}$ with a primary polyclonal antiserum directed against human Dkk-3 (goat-anti-human Dkk-3 polyclonal $1.5 \mu \mathrm{g} / \mathrm{mL}$; R\&D Systems Inc., Minneapolis, USA). Primary antiserum was detected by incubation with a biotinylated secondary antibody (biotinylated horse-anti-goat IgG, Vector Laboratories Inc., Burlingame, CA, USA) with the Vectastain Elite ABC Kit (Vector Laboratories Inc., Burlingame, CA, USA) and the FAST DAB Tablet Set (Sigma, Vienna, Austria). The sections were washed with water, counterstained with Mayer's hematoxylin (Merck, Darmstadt, Germany) and mounted with medite Pertex ${ }^{\circledR}$ (Medite Medizintechnik, Burgdorf, Germany). Specificity of antiserum against human Dkk-3 was proven by blocking experiments with an excess of recombinant human Dkk-3. Simultaneously, a second TMA section from each specimen was stained with CD31 to identify microvessels by immunostaining endothelial cells for CD31 antigen. CD31 was detected with a monoclonal mouse anti-human CD31 antibody diluted 1:40 (clone JC70A, Dako Cytomation, Copenhagen, Denmark) and the respective secondary antibody (biotinylated goat antimouse IgG, Vector Laboratories Inc., Burlingame, CA, USA).

\subsection{Quantification of neoangiogenesis}

All CD31-positive stained microvessels from cancer tissue and adjacent non-cancerous tissue samples were manually counted by two independent investigators (P.M. and A.A.); the whole sample was counted at X400 magnification. Any stained endothelial cell or cell-cluster was identified as an independent vessel, if these cells or cell-clusters were clearly separate from each other. The average count of both investigators was defined as "microvessel count per sample". Additionally, samples were grouped according to their microvessel count: $<10$ microvessels per sample, $\geqslant 10<20$ microvessels per sample and $\geqslant 20$ microvessels per sample.

\subsection{Evaluation of Dkk-3 expression}

Two independent pathologists (P.M. and S.S.) with no prior knowledge of the clinical data analyzed TMA sections in parallel.

A sample was considered as Dkk-3 positive if more than $90 \%$ of the identified microvessels were stained, irrespective of staining intensity. A sample was considered as Dkk-3 negative if less than $10 \%$ of the identified microvessels were stained. If a sample showed Dkk-3 staining in $10 \%$ to $90 \%$ of the identified microvessels it was defined as intermediate staining.

\subsection{Statistical analysis}

Statistical analysis was performed with SPSS 14.0 (SPSS, Inc., Chicago, IL). Descriptive statistics for continuous measures (microvessel count) are given as the mean with the respective $95 \%$ confidence interval (CI) in parenthesis. For discrete data (Dkk-3 expression) frequency counts and percentages were tabulated. Results of the various estimates were compared using either the chi-squared test or One-way ANOVA. All data on the date and cause of death were confirmed by the "Tumorregister Tirol", a register kept by the Tyrolean government. Cumulative patient survival was estimated using the Kaplan-Meier method; for comparison of survival curves the log-rank test (Mantel-Haenszel method) was used. The Cox proportional hazards linear regression model was computed to determine in a forward stepwise procedure which factors were associated simultaneously with survival. Results were considered significant when $P<0.05$.

\section{Results}

\subsection{Expression of Dkk-3 protein in colorectal cancer tissue}

None of the cancer samples showed Dkk-3 staining in colorectal carcinoma cells, either in cellular membrane, cytoplasm or in the nucleus (Fig. 1B).

Out of all cancer samples $(n=403), 63.8 \%$ (257/403) presented with CD31-positive microvessels (Fig. 1A), 36.2\% (146/403) had no microvessels. With regard to samples with microvessels, the mean microvessel count per sample was 5.59 vessels (95\% CI: 4.92-6.26). Three groups were formed according to microvessel count (see "Materials and Methods"): less than 10 microvessels per sample were observed in 
A

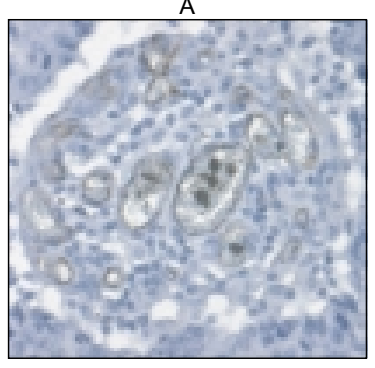

C

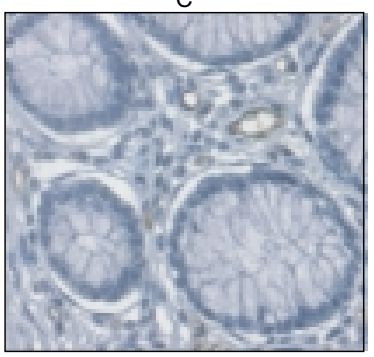

$E$

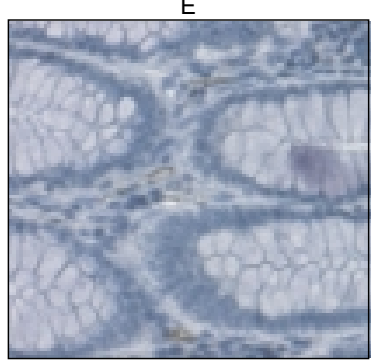

Fig. 1. CD31 and Dkk-3 expression in colorectal cancer tissue (A, $\mathrm{B})$, colorectal adjacent non-cancerous tissue (C, D), and normal colorectal mucosa (E, F). A (CD31), B (Dkk-3): colorectal cancer tissue; C (CD31), D (Dkk-3): colorectal adjacent non-cancerous tissue; $\mathrm{E}$ (CD31), F (Dkk-3): normal colorectal mucosa.

$73.5 \%(189 / 257), \geqslant 10$ but $<20$ microvessels in $17.5 \%$ $(45 / 257)$ and $\geqslant 20$ microvessels in $9 \%(23 / 257)$.

A positive staining reaction for Dkk-3 (Fig. 2) was found in $67.7 \%$ of samples with CD31-positive microvessels (174/257). No sample showed intermediate staining; and $32.3 \%$ (83/257) were Dkk-3 negative (Fig. 2). In terms of 174 Dkk-3 positive samples, $170(97.7 \%)$ showed Dkk-3 expression in $100 \%$ of microvessels (Fig. 1B), four (2.3\%) samples revealed Dkk-3 expression in more than $90 \%$ of microvessels. In all Dkk-3 negative samples $0 \%$ of identified microvessels were stained.

Furthermore, Dkk-3-positive samples showed a statistically significantly higher mean microvessel count [9.70 vessels (95\% CI: 8.52-10.87)] than did Dkk-3negative samples [6.82 vessels (95\% CI: 6.34-7.30); $F=10.7, D F=1, P=0.001]$. Moreover, Dkk-3 expression within the three microvessel count groups revealed a statistically significant increase with rising numbers of intratumoral microvessels per sample ( $\chi^{2}=450.1, D F=6, P<0.0001$; Fig. 3A).

Univariate analysis of clinicopathologic variables with regard to Dkk-3 expression is shown in Table 1. Statistically significant differences were observed for tumor site $\left(\chi^{2}=7.9, D F=2, P=0.02\right)$ and patient age $(F=4.1, D F=2, P=0.018)$. A borderline correlation was found with pT stage $(P=0.054)$, tumor differentiation $(P=0.079)$ and tumor progression $(P=0.089)$, respectively.

Survival analysis according to the Kaplan-Meier method showed a trend toward improved disease-free survival for patients with Dkk-3-negative samples (logrank: $5.7, D F=2, P=0.057)$. Within this group, the five-year disease-free survival rate was $70 \%$ as compared with $59 \%$ for patients with Dkk-3-positive samples. Univariate survival analysis revealed Tstage, nodal status, metastases and tumor differentiation as significant predictors of disease-free survival (Table 2). Regarding overall survival, patients with Dkk-3-positive samples and Dkk-3-negative samples were comparable $(p=0.175)$. Multivariate diseasefree survival analysis showed nodal status and metastases as independent prognostic parameters (Table 3).

\subsection{Expression of Dkk-3 protein in colorectal adjacent non-cancerous tissue}

None of the adjacent non-cancerous tissue samples showed Dkk-3 staining in the glandular epithelium, either in cellular membrane, cytoplasm or in the nucleus (Fig. 1D).

CD31-positive microvessels were found in $84.3 \%$ (268/318) of adjacent non-cancerous tissue samples (Fig. 1C). The mean microvessel count per sample was 9.33 vessels (95\% CI: 8.36-10.30). Again, three groups were formed according to microvessel count: less than 10 microvessels per sample were identified in $65.5 \%$ $(176 / 268), \geqslant 10$ but $<20$ microvessels in $18 \%(48 / 268)$ and $\geqslant 20$ microvessels in $16.5 \%(45 / 268)$. Fifty samples $(15.7 \%)$ contained no microvessels.

In terms of CD31-positive samples, 56\% (150/268; Fig. 2) showed a positive staining reaction for Dkk-3 in all microvessels (Fig. 1D), and 44\% (118/268; Fig. 2) were negative for Dkk-3 in all microvessels. No sample showed intermediate staining.

Dkk-3-positive samples showed a statistically significantly higher mean microvessel count [14.51 (95\% CI: 12.88-16.14)] than did Dkk-3-negative samples [6.64 (95\% CI: 6.40-6.89); $F=93.2, D F=2, P<$ 


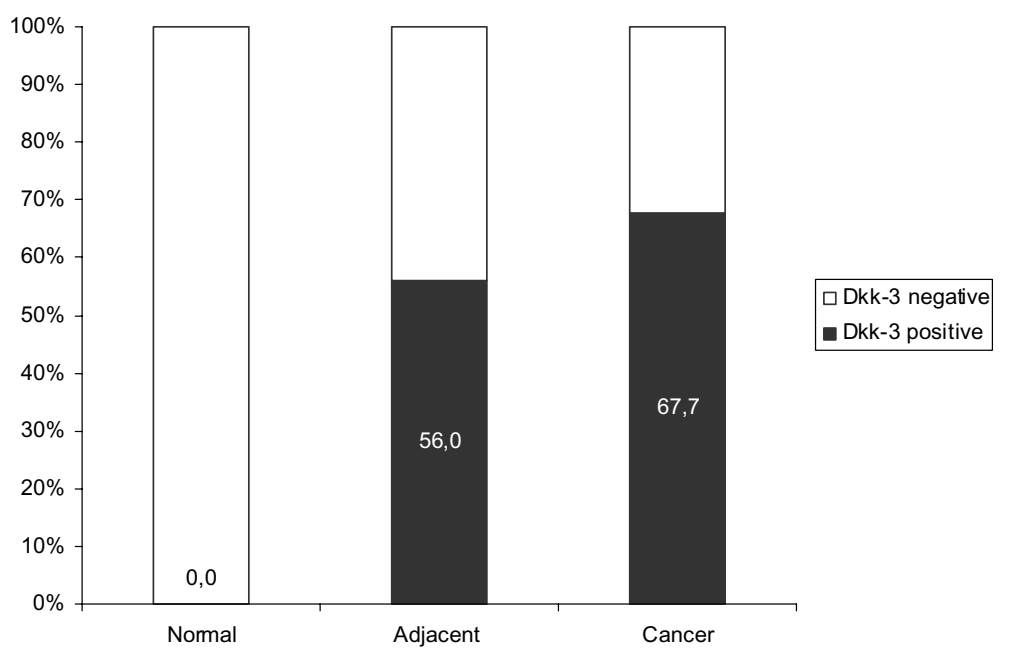

Fig. 2. Proportion of Dkk-3-positive and Dkk-3-negative samples in normal colorectal mucosa, colorectal adjacent non-cancerous tissue and colorectal cancer tissue.

0.0001]. In addition, Dkk-3 expression in the three microvessel count groups revealed a statistically significant increase with rising numbers of microvessels per sample $\left(\chi^{2}=430.7, D F=6, P<0.0001\right.$; Fig. 3B).

There were no significant correlations between Dkk3 expression in adjacent non-cancerous tissue and the clinicopathologic variables described above.

\subsection{Expression of Dkk-3 protein in normal colorectal тисова}

None of the 127 normal colorectal mucosal tissues showed a positive staining reaction for Dkk-3 protein, either in the CD31-positive endothelial cells of the microvessels or in the epithelial cells of the mucosa (Fig. 1. E, F and Fig. 2).

\section{Discussion}

In contrast to Dkk-1, Dkk-2, and Dkk-4, which have the ability to modulate the WNT/beta-catenin signaling pathway [10], the biological role of Dkk-3 remains unclear. However, Dkk-3 has been proposed to act as a suppressor of human tumor growth because it is downregulated in various types of cancer or tumor cell lines and its overexpression is associated with inhibition of cell proliferation [12-20]. With regard to colorectal cancer or colorectal tumor cell lines, no data are available on whether Dkk-3 is downregulated or upregulated. Byun et al. reported that there are no differences between Dkk-3 expression in colorectal cancer and in matched normal colon tissue. In that study Dkk-3 was detected by in situ RNA hybridization in five of 14 normal colon tissues and four of 15 malignant colon tissue samples [21]. In the present study, which is the first analysis of Dkk-3 at the protein level in colorectal cancer, none of the 403 tumor samples showed Dkk-3 staining in colorectal carcinoma cells, either in cellular membrane, cytoplasm or in the nucleus. Furthermore, there was no positive staining reaction for Dkk-3 protein in the glandular epithelium of 127 normal colorectal mucosa samples. In comparison to other malignancies, Dkk-3 can be assumed to play a different role in colorectal tumor growth. For this reason, our study was designed to investigate the relationship between Dkk-3 and neoangiogenesis in colorectal cancer.

Angiogenesis is the driving force behind tumor growth and therefore one of the strongest features of prognostic relevance in cancer identified so far. Although angiogenesis initially develops by incorporating host blood vessels, solid tumors apparently cannot grow more than $1.0 \mathrm{~mm}^{3}$ unless the tumor synthesizes its own new networks of morphologically and functionally abnormal microvessels [22,23]. This process of neovascularization requires a direct or indirect role of angiogenic factors $[24,25]$ that are derived mainly from cancer cells and stimulate pre-existing host capillaries to induce endothelial cell proliferation, migration and, eventually, maturation into tube-like structures [26]. St. Croix et al. compared gene expression patterns of endothelial cells from blood vessels of normal and malignant colorectal tissues and revealed 46 tumor endothelial markers (TEMs) that were expressed at sub- 

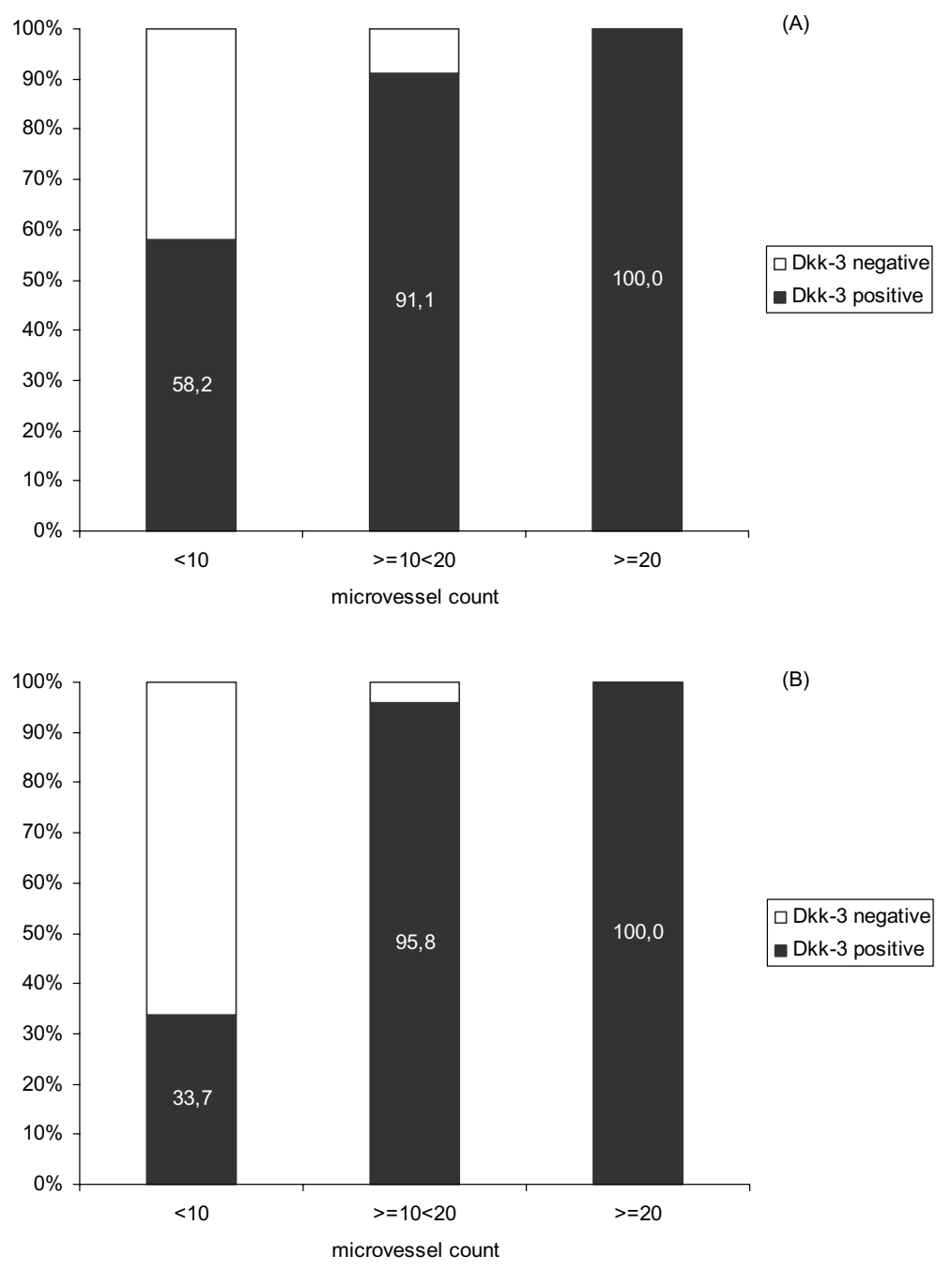

Fig. 3. Dkk-3 expression in colorectal cancer tissue $\underline{3 \mathrm{~A}} ; P<0.0001)$ and colorectal adjacent non-cancerous tissue $\underline{(3 \mathrm{~B}} ; P<0.0001)$ in the three microvessel count groups.

stantially higher levels ( $>10$-fold) in tumor-associated endothelium [8]. Although that study showed that gene expression of Dkk-3 - as one of these TEMs - is strongly upregulated in colorectal cancer endothelium, further analyses were not performed. To our knowledge, the present study is the first investigation of Dkk-3 protein expression in tumor endothelium of colorectal cancer tissue versus colorectal adjacent non-cancerous tissue and normal colorectal mucosa. This study demonstrates that $174(67.7 \%)$ out of 257 colorectal cancer samples with CD31-positive microvessels had a positive staining reaction for Dkk-3 in endothelial cells of all microvessels, whereas in samples of normal colorectal mucosa all CD31-positive microvessels lacked Dkk3 staining. According to St. Croix's Science study indicating that tumor and normal endothelium are distinct at the molecular level [8], our analysis confirms these differences at the protein level. In addition, Dkk3-positive colorectal cancer samples showed a statistically significantly higher mean microvessel count $(9.70$ vessels) than did Dkk-3-negative samples (6.82 vessels; $P=0.001)$. Furthermore, it must be emphasized that the positivity rate of Dkk-3 expression increases in parallel with the microvessel count per sample $(P<$ 0.0001). From these cardinal findings we assume that Dkk-3 is a pro-angiogenic factor involved in neovascularization during tumor growth. Correlating Dkk-3 expression with several clinicopathological parameters including survival showed some absolutely remarkable associations, for example improved disease-free sur- 
Table 1

Dkk-3 expression in colorectal cancer tissue and clinicopathologic variables $(n=403)$

\begin{tabular}{|c|c|c|c|c|c|}
\hline Variables & All cases $(n)$ & $\begin{array}{c}\text { Dkk-3-positive } \\
(n=174,43.1 \%)\end{array}$ & $\begin{array}{c}\text { Dkk-3-negative } \\
(n=83,20.6 \%)\end{array}$ & $\begin{array}{c}\text { No microvessels } \\
(n=146,36.2 \%)\end{array}$ & Significance $(\mathrm{P})$ \\
\hline Gender & 403 & & & & \\
\hline Male & 224 & $106(47.3)$ & $41(18.3)$ & $77(34.4)$ & \\
\hline Female & 179 & $68(38.0)$ & $42(23.3)$ & $69(38.5)$ & 0.152 \\
\hline Mean age $(95 \% \mathrm{CI})$ & $69.1(67.9-70.2)$ & $68.0(66.3-69.8)$ & $67.2(64.4-70.1)$ & $71.3(69.36-73.2)$ & 0.018 \\
\hline Tumor site & 403 & & & & \\
\hline Colon & 260 & $105(40.3)$ & $48(18.5)$ & $107(41.2)$ & \\
\hline Rectum & 143 & $69(48.3)$ & $35(24.5)$ & $39(27.2)$ & 0.020 \\
\hline Tumor differentiation & 403 & & & & \\
\hline Grade $1 / 2$ & 332 & $149(44.0)$ & $71(21.4)$ & $112(33.7)$ & \\
\hline Grade 3/4 & 71 & $25(35.2$ & $12(16.9)$ & 34 (47.9) & 0.079 \\
\hline pT stage & 403 & & & & \\
\hline pT1 & 37 & $15(40.5)$ & $13(35.1)$ & $9(24.4)$ & \\
\hline pT2 & 73 & $39(53.4)$ & $12(16.4)$ & $22(30.2)$ & \\
\hline pT3 & 247 & $105(42.5)$ & $50(20.2)$ & $92(37.3)$ & \\
\hline pT4 & 46 & $15(32.6)$ & $8(17.4)$ & $23(50.0)$ & 0.054 \\
\hline $\mathrm{pN}$ stage & 387 & & & & \\
\hline $\mathrm{pN}-$ & 192 & $91(47.4)$ & $40(20.8)$ & $61(31.8)$ & \\
\hline $\mathrm{pN}+$ & 195 & $79(40.5)$ & $39(20.0)$ & $77(39.5)$ & 0.260 \\
\hline M stage & 403 & & & & \\
\hline M0 & 319 & 137 (42.9) & $71(22.3)$ & $111(34.8)$ & \\
\hline M1 & 84 & $37(44.0)$ & $12(14.3)$ & $35(41.7)$ & 0.230 \\
\hline Tumor progression & 403 & & & & \\
\hline No & 260 & $109(42.0)$ & $62(23.8)$ & $89(34.2)$ & \\
\hline Yes & 143 & $65(45.5)$ & $21(14.7)$ & $57(39.8)$ & 0.089 \\
\hline
\end{tabular}

Table 2

Univariate analysis of disease-free survival (colorectal cancer tissue; $n=403$ )

\begin{tabular}{lrrc}
\hline Variable & \multicolumn{1}{c}{$\chi^{2}$} & DF & \multicolumn{1}{c}{$\mathrm{P}$} \\
\hline Gender (Male vs. Female) & 1.06 & 1 & n.s. \\
Age (continuous) & 0.45 & 1 & n.s. \\
Tumor site (Colon vs. Rectum) & 0.30 & 1 & n.s. \\
Tumor differentiation (G1/G2 vs. G3/G4) & 12.21 & 1 & 0.000 \\
pT stage (pT1 vs. pT2 vs. pT3 vs. pT4) & 40.38 & 3 & 0.000 \\
pN stage (pN- vs. pN+) & 72.83 & 1 & 0.000 \\
M stage (M0 vs. M1) & 357.11 & 1 & 0.000 \\
Dkk-3 (Dkk-3-pos. vs. Dkk-3-neg. vs. no microvessels) & 5.73 & 2 & 0.057 \\
\hline
\end{tabular}

Table 3

Mulitvariate analysis of disease-free survival (colorectal cancer tissue; $n=403$ )

\begin{tabular}{|c|c|c|c|c|c|}
\hline \multirow{2}{*}{\multicolumn{2}{|c|}{ Variable }} & \multirow[t]{2}{*}{ RR } & \multicolumn{2}{|c|}{$95 \% \mathrm{CI}$ for mean } & \multirow[t]{2}{*}{$\mathrm{P}$} \\
\hline & & & Lower bound & Upper bound & \\
\hline \multirow{2}{*}{\multicolumn{2}{|c|}{$\begin{array}{l}\mathrm{pN} \text { stage }(\mathrm{pN}-\mathrm{vs} . \mathrm{pN}+) \\
\mathrm{M} \text { stage }(\mathrm{M} 0 \text { vs. } \mathrm{M} 1)\end{array}$}} & 2.21 & 1.41 & 3.47 & 0.001 \\
\hline & & 13.13 & 8.38 & 20.55 & 0.000 \\
\hline \multirow[t]{3}{*}{ Dkk-3 } & no microvessels & & & & 0.69 \\
\hline & Dkk-3-neg & 1.21 & 0.73 & 2.02 & 0.46 \\
\hline & Dkk-3-pos & 1.06 & 0.64 & 1.76 & 0.82 \\
\hline
\end{tabular}

$R R \ldots$ relative risk; $C I \ldots$ confidence interval.

vival for patients with Dkk-3-negative samples $(P=$ 0.057). Nevertheless, it is difficult to interpret these clinical results because of the significant proportion of samples without microvessels $(n=146 ; 36.2 \%)$.

It is widely accepted that the "angiogenic switch" is "on" if the balance between pro-angiogenic and antiangiogenic factors is tipped in favor of angiogene- sis [27,28]. Pro- and anti-angiogenic molecules can emanate from cancer cells, cancer stromal cells, blood and the extracellular matrix [29]. Sugiyama et al. used laser capture microdissection in tissue samples from 11 patients undergoing colorectal resection for cancer to isolate pure populations of cancer cells, cancer stromal cells (including vascular endothelial cells) and corre- 
sponding adjacent normal epithelial and stromal cells for gene expression arrays. They demonstrated more genes contributing to angiogenesis in cancer cells than in adjacent normal epithelial cells and more in cancer stromal cells than in adjacent normal stromal cells. In addition, expression of the angiogenesis-related proteins VEGF and thrombospondin 2 was determined using immunohistochemistry. Both proteins were stained strongly positive to positive in cancer cells and cancer stromal cells, but also positive in adjacent normal stromal cells [30]. Our study illustrates that colorectal adjacent non-cancerous tissue is similar in comparison to colorectal cancer tissue with regard to Dkk-3 protein expression: 1) We found no positive staining reaction for Dkk-3 in the glandular epithelium of 318 colorectal adjacent non-cancerous tissue samples; 2) a positive staining reaction for Dkk-3 was seen in endothelial cells of all microvessels in 150/268 (56\%) CD31positive samples; 3 ) a significantly higher mean microvessel count (14.51 vessels) was shown in Dkk-3positive samples than in Dkk-3-negative samples (6.64 vessels; $P<0.0001)$; 4) Dkk-3 expression increased with increasing number of microvessels per sample $(P<0.0001)$. These results support the concept that carcinogenesis is a multicellular process in which alteration of the surrounding microenvironment is required for tumor progression [31,32]. In our opinion, scientific focus must return to the "entire" tumor, which includes the epithelial cancer cells, the surrounding cancer stromal cells, but also the adjacent non-cancerous tissue with its altered vascular endothelial cells or abnormal microvessels. As a result of various angiogenic factors secreted mainly by cancer cells and tumor-associated stromal cells, more than half of colorectal carcinomas are characterized by intensified angiogenic activity, a feature related to unfavorable outcome [33].

In conclusion, our study demonstrates for the first time that microvessels of colorectal cancer and adjacent non-cancerous tissue are similar with regard to Dkk-3 protein expression, but distinct from normal colorectal mucosa. Therefore, Dkk-3 can be considered a putative pro-angiogenic protein in the neovascularization process. It may have the potential to serve as a marker for neoangiogenesis and might be a target structure for novel therapeutic approaches. Nevertheless, it is mandatory that the findings presented here be confirmed with routinely processed tissue sections.

\section{Acknowledgments}

We thank Ines Tschoerner, Mag. Daniela Pirkebner and Martina Zimmermann for their excellent technical assistance.

\section{References}

[1] E. Sperti, R. Faggiuolo, A. Gerbino et al., Outcome of Metastatic Colorectal Cancer: Analysis of a Consecutive Series of 229 Patients. The Impact of a Multidisciplinary Approach, Dis Colon Rectum (2006), Epub ahead of print.

[2] G.B. Secco, R. Fardelli, D. Gianquinto et al., Efficacy and cost of risk-adapted follow-up in patients after colorectal cancer surgery: a prospective, randomized and controlled trial, Eur J Surg Oncol 28 (2002), 418-423.

[3] M. Zitt, G. Mühlmann, H. Weiss et al., Assessment of riskindependent follow-up to detect asymptomatic recurrence after curative resection of colorectal cancer, Langenbecks Arch Surg 391 (2006), 369-375.

[4] J. Folkman, Tumor angiogenesis: therapeutic implications, $N$ Engl J Med 285 (1971), 1182-1186.

[5] D. Hanahan and J. Folkman, Patterns of emerging mechanisms of the angiogenic switch during tumorigenesis, Cell 86 (1996), 353-364.

[6] R. Poon Tung-Ping, Fan Sheung-Tat and J. Wong, Clinical significance of angiogenesis in gastrointestinal cancers, Ann Surg 238 (2003), 9-28.

[7] P. Carmeliet and R.K. Jain, Angiogenesis in cancer and other diseases, Nature 407 (2000), 249-257.

[8] B. St. Croix, C. Rago, V. Velculescu et al., Genes expressed in human tumor endothelium, Science 289 (2000), 1197-1202.

[9] V.E. Krupnik, J.D. Sharp, C. Jiang et al., Functional and structural diversity of the human Dickkopf gene family, Gene 238 (1999), 301-313.

[10] C. Niehrs, Function and biological roles of the Dickkopf family of WNT modulators, Oncogene 25 (2006), 7469-7481.

[11] B. Mao, W. Wu, G. Davidson et al., Kremen proteins are Dickkopf receptors that regulate WNT/beta-catenin signalling, Nature 417 (2002), 664-667.

[12] T. Tsuji, M. Miyazaki, M. Sakaguchi, Y. Inoue and M. Namba, A REIC gene shows down-regulation in human immortalized cells and human tumor-derived cell lines, Biochem Biophys Res Commun 268 (2000), 20-24.

[13] K. Kobayashi, M. Ouchida, T. Tsuji et al., Reduced expression of the REIC/Dkk-3 gene by promoter-hypermethylation in human tumor cells, Gene 282 (2002), 151-158.

[14] I. Nozaki, T. Tsuji, O. Iijima et al., Reduced expression of REIC/Dkk-3 gene in non-small cell lung cancer, Int J Oncol 19 (2001), 117-121.

[15] K. Kurose, M. Sakaguchi, Y. Nasu et al., Decreased expression of REIC/Dkk-3 in human renal clear cell carcinoma, $J$ Urol 171 (2004), 1314-1318.

[16] J. Roman-Gomez, A. Jimenez-Velasco, X. Agirre et al., Transcriptional silencing of the Dickkopfs-3 (Dkk-3) gene by CpG hypermethylation in acute lymphoblastic leukaemia, Br J Cancer 91 (2004), 707-713.

[17] S.Y. Hsieh, P.S. Hsieh, C.T. Chiu and W.Y. Chen, Dickkopf3/REIC functions as a suppressor gene of tumor growth, Oncogene 23 (2004), 9183-9189. 
[18] Y. Kawano, M. Kitaoka, Y. Hamada, M.M. Walker, J. Waxman and R.M. Kypta, Regulation of prostate cell growth and morphogenesis by Dickkopf-3, Oncogene 25 (2006), 6528-6537.

[19] D. Lodygin, A. Epanchintsev, A. Menssen, J. Diebold and H. Hermeking, Functional epigenomics identifies genes frequently silenced in prostate cancer, Cancer Res 65 (2005), 4218-4227.

[20] S. Kuphal, S. Lodermeyer, F. Bataille, M. Schuierer, B.H. Hoang and A.K. Bosserhoff, Expression of Dickkopf genes is strongly reduced in malignant melanoma, Oncogene $\mathbf{2 5}$ (2006), 5027-5036.

[21] T. Byun, M. Karimi, J.L. Marsh, T. Milovanovic, F. Lin and R.F. Holcombe, Expression of secreted WNT antagonists in gastrointestinal tissues: potential role in stem cell homeostasis, J Clin Pathol 58 (2005), 515-519.

[22] J. Denekamp, Angiogenesis, neovascular proliferation and vascular pathophysiology as targets for cancer therapy, $\mathrm{Br} \mathrm{J}$ Radiol 66 (1993), 181-196.

[23] P. Vaupel, F. Kallinowski and P. Okunieff, Blood flow, oxygen and nutrient supply, and metabolic microenvironment of human tumours: a review, Cancer Res 49 (1989), 6449-6465.

[24] M. Klagsbrun and P.A. D'Amore, Regulators of angiogenesis, Annu Rev Physiol 53 (1991), 217-239.

[25] D. Zagzag, Angiogenic growth factors in neural angiogenesis and neoplasia, Am J Pathol 146 (1995), 293-309.
[26] R.K. Jain, Molecular regulation of vessel maturation, Nat Med 9 (2003), 685-693.

[27] D. Hanahan and R.A. Weinberg, The hallmarks of cancer, Cell 100 (2000), 57-70.

[28] N. Bouck, V. Stellmach and S.C. Hsu, How tumors become angiogenic, Adv Cancer Res 69 (1996), 135-174.

[29] D. Fukumura, R. Xavier and T. Sugiura, Tumor induction of VEGF promoter activity in stromal cells, Cell 94 (1998), $712-725$.

[30] Y. Sugiyama, B. Farrow, C. Murillo, J. Li, H. Watanabe, K. Sugiyama and M. Evers, Gastroenterology 128 (2005), 480486.

[31] K. Birkenkamp-Demtroder, L.L. Christensen, S.H. Olesen et al., Gene expression in colorectal cancer, Cancer Res 62 (2002), 4352-4363.

[32] Y.M. Lin, Y. Furukawa, T. Tsunoda, C.T. Yue, K.C. Yang and Y. Nakamura, Molecular diagnosis of colorectal tumors by expression profiles of 50 genes expressed differentially in adenomas and carcinomas, Oncogene 21 (2002), 4120-4128.

[33] A. Giatromanolaki, E. Sivridis and M.I. Koukourakis, Angiogenesis in Colorectal Cancer: Prognostic and Therapeutic Implications, Am J Clin Oncol 29 (2006), 408-417. 


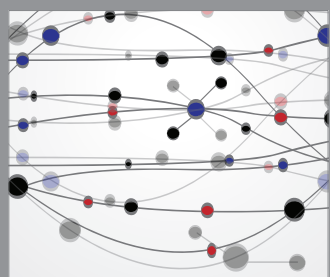

The Scientific World Journal
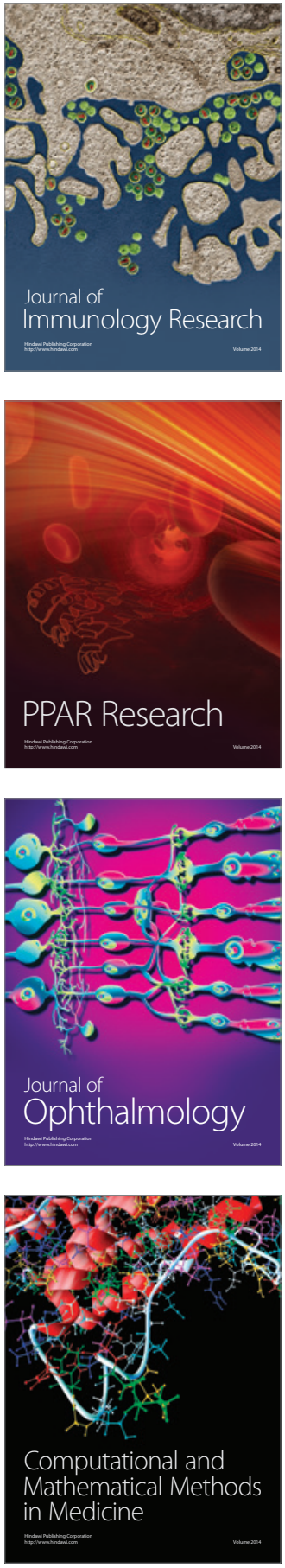

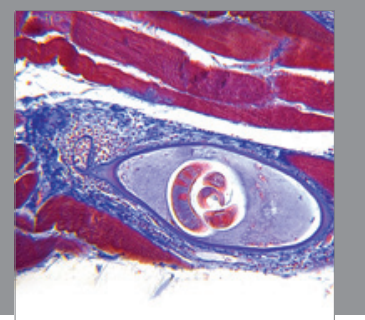

Gastroenterology

Research and Practice
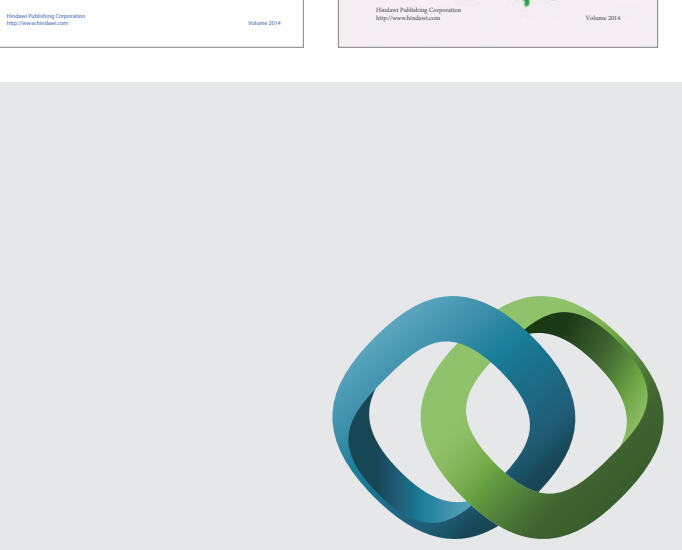

\section{Hindawi}

Submit your manuscripts at

http://www.hindawi.com
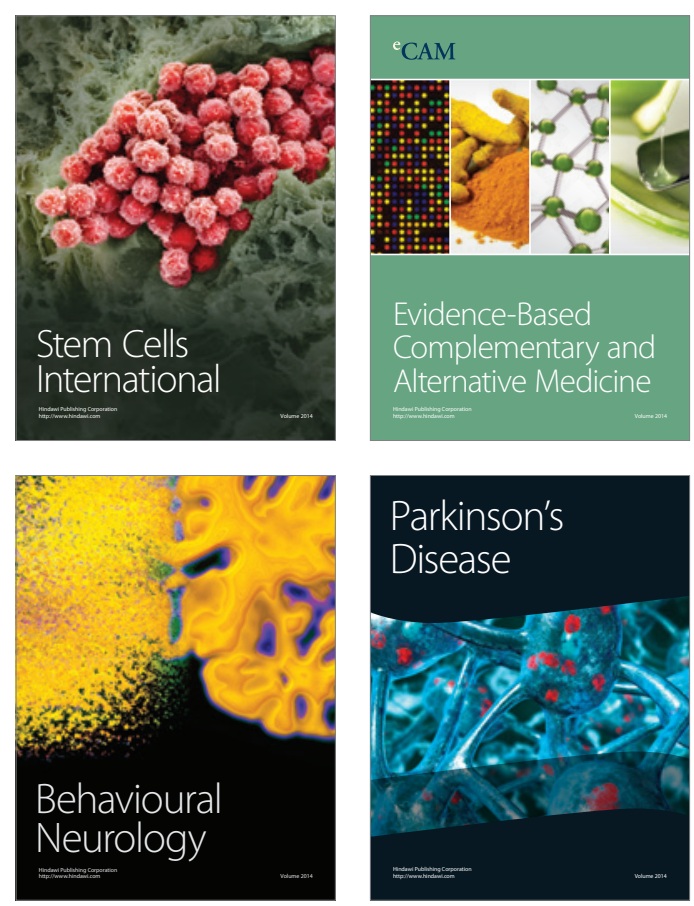

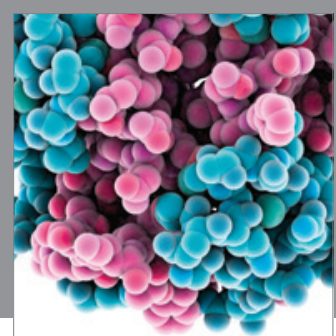

Journal of
Diabetes Research

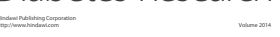

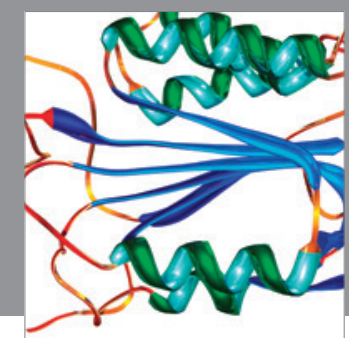

Disease Markers
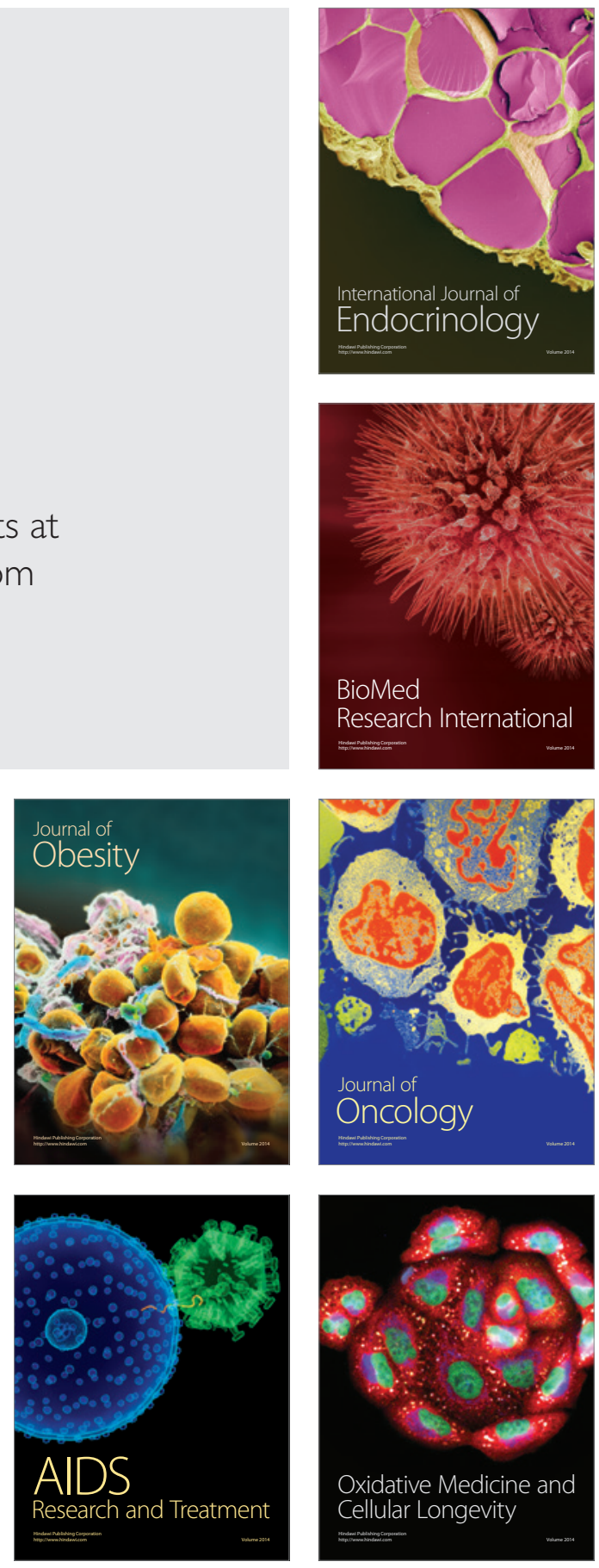